\title{
Experimental and calculated nuclear reaction data relevant to innovative production of medical radioisotopes
}

\author{
E. Menapace ${ }^{1, a, b}$, M.L. Bonardi ${ }^{1}$, F. Groppi ${ }^{1}$, E. Persico ${ }^{1}$, and Z.B. Alfassi ${ }^{2}$ \\ 1 Università degli Studi di Milano and INFN-LASA Laboratory, via F.lli Cervi 201, 20090 Segrate, Milano, Italy \\ 2 Department of Nuclear Engineering, Ben Gurion University, Beer Sheva, Israel
}

\begin{abstract}
Selected results are presented and discussed concerning nuclear data relevant to the production of innovative medical radionuclides of special interest, as from research activities brought up at INFN LASA Laboratory, with reference to irradiation experiments at the $\mathrm{K}=38$ Cyclotron JRC-Ispra Cyclotron Laboratory. Measurements of excitation functions of the involved nuclear reactions are considered, in comparison with corresponding theoretical calculations, especially including radiation detection measurements, through the relevant instruments, and the requested radiochemistry studies. The considered radionuclides and the relevant production reactions are specified in the following introduction, also concerning recent and new measurements.
\end{abstract}

\section{Introduction}

A series of high specific activity accelerator-produced radionuclides in no-carrier-added (NCA) form, for uses in therapeutic applications and in related PET and SPECT diagnoses have been critically investigated and/or fully revisited, including thick target measurements, particularly:

1. NCA ${ }^{64} \mathrm{Cu}$, produced by the ${ }^{n a t} \mathrm{Zn}(\mathrm{d}, \alpha \times \mathrm{xn})$ and ${ }^{\text {nat }} \mathrm{Zn}(\mathrm{d}, 2 \mathrm{pxn})$ reactions for simultaneous $\beta^{+} / \beta^{-}$metabolic radiotherapy with intrinsic PET imaging, including the short-lived radionuclide ${ }^{61} \mathrm{Cu}$, and also ${ }^{66,67} \mathrm{Ga}$ as highly relevant radionuclides;

2. ${ }^{186 \mathrm{~g}} \mathrm{Re}$, produced by the ${ }^{186} \mathrm{~W}(\mathrm{p}, \mathrm{n})$ reaction for bone metastases pain palliation by negatron $(1.1 \mathrm{MeV})$ metabolic radiotherapy including SPECT imaging;

3. $\mathrm{NCA}{ }^{211} \mathrm{At} /{ }^{211 \mathrm{~g}} \mathrm{Po}$, produced by the ${ }^{209} \mathrm{Bi}(\alpha, 2 \mathrm{n})$ reaction, with internal spike of gamma emitter ${ }^{210} \mathrm{At}$ from the ${ }^{209} \mathrm{Bi}(\alpha, 3 \mathrm{n})$ reaction (and small amount of ${ }^{210} \mathrm{Po}$ as radiotoxic long-lived impurity), for high-LET radio- and immuno-radiotherapy;

4. ${ }^{103} \mathrm{Pd}$, as a commonly used radionuclide for therapeutic treatments, produced by the alternative ${ }^{103} \mathrm{Rh}(\mathrm{d}, 2 \mathrm{n})$ reaction route;

5. Studies intended to the production of therapeutic ${ }^{230} \mathrm{~Pa} /{ }^{230} \mathrm{U}$ alpha-emitters by the reaction ${ }^{232} \mathrm{Th}(\mathrm{p}, 3 \mathrm{n})$.

The nuclear data relevant to the excitation functions of the involved nuclear reactions, including the ones concerning the production of radionuclides as contaminants, are discussed through appropriate comparison of present and other available and critically selected experimental values previously published and with model calculations presently done [1-3]. Particularly in the following, special care is devoted to the discussion of the results for the production of ${ }^{211} \mathrm{At}$, ${ }^{210} \mathrm{At},{ }^{186 \mathrm{~g}} \mathrm{Re},{ }^{103} \mathrm{Pd}$ and ${ }^{230} \mathrm{~Pa} /{ }^{230} \mathrm{U}$ radionuclides for which

\footnotetext{
${ }^{a}$ Present author, e-mail: enzo.menapace@unibo.it

${ }^{b}$ Present address, Università degli Studi di Milano and INFNLASA, Milano, Italy
}

measured and calculated excitation functions are compared and discussed with previously existing ones [8-13].

For the other radionuclides, main care has been devoted by the authors to critically survey the relevant results, as from the published papers [3-7].

\section{Materials and experimental methods}

Targets were irradiated at the Scanditronix MC40 cyclotron $(\mathrm{K}=38)$, JRC-Ispra (Varese, Italy) of the EC, that can deliver variable energy proton or alpha beams with energies up to $38 \mathrm{MeV}$ and deuteron beams up to $19 \mathrm{MeV}$.

Concerning the At radionuclides (RNs), ${ }^{211}$ At is clearly the most promising for labelling drugs and radiopharmaceutical compounds for metabolic radionuclide therapy, for the following reasons:

- the half-life of $7.214 \mathrm{~h}$ is reasonably sufficient long to label organic compounds, due to the strengths of C-At, O-At and $\mathrm{N}$-At bonds, that are similar to those of iodine;

- the ${ }^{211}$ At $\alpha$ branching of $41.80 \%$, which is the highest among the "medium-lived" At RNs. Although decaying by EC, the further $58.20 \%$ also leads to alpha emission through ${ }^{211 \mathrm{~g}} \mathrm{Po}$ that has an $\alpha$-branching of $100 \%$ and an half-life of $516 \mathrm{~ms}$ too short to allow its migration from the location of its formation. As a consequence the "overall" $\alpha$-branching of the decay of ${ }^{211}$ At is $100 \%$;

- the energy of the $\alpha$-particles ranges from $5870 \mathrm{keV}$ to $7450 \mathrm{keV}$ (with an approximated arithmetical average of $6665 \mathrm{keV})$;

- the two main $\alpha$-particles of the ${ }^{211} \mathrm{At} \rightarrow{ }^{211 \mathrm{~g}}$ Po decay have an average range of 60 to $67 \mu \mathrm{m}$ in water and soft tissues and a nearly optimal $\mathrm{LET}_{\infty}$ of $100-130 \mathrm{eV} \cdot \mathrm{nm}^{-1}$, which is around the maximum of the Q-curve for energetic ions.

A good choice for a production method of ${ }^{211}$ At has been to minimize isotopic contamination by the Po nuclides to negligible levels. The direct production method based on the nuclear reactions ${ }^{209} \mathrm{Bi}(\alpha, 2 \mathrm{n})^{211}$ At seems the most satisfactory, because it can be done in a medium energy cyclotron, 


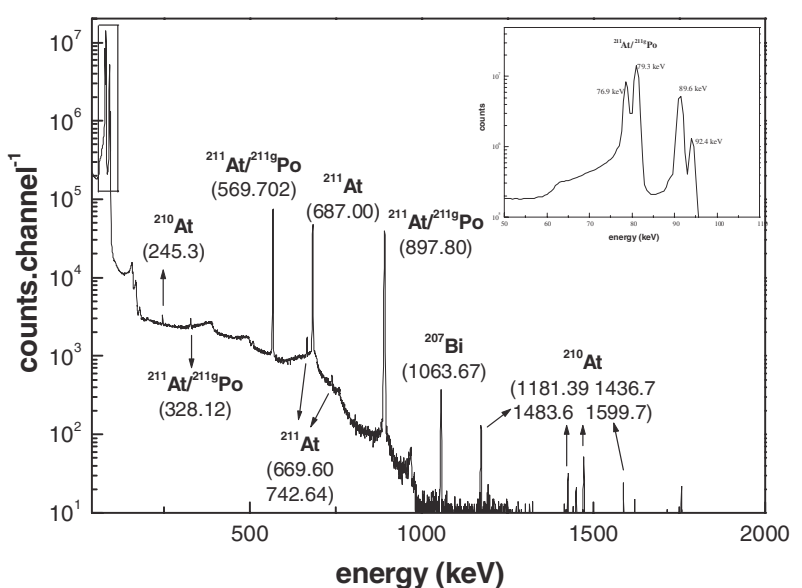

Fig. 1. $\gamma$-spectrum obtained with a HPGe detector from a Bi target irradiated with $28.8 \mathrm{MeV} \alpha$-particles. In the zoom region, Po X-ray emissions are evidenced.

leading to a high yield and low contamination by the only radionuclidic impurity ${ }^{210} \mathrm{At}$, that can be assumed as internal spike, or not produced if the $\alpha$ beam energy is kept less than $28.61 \mathrm{MeV}$.

The irradiation experiments for ${ }^{211}$ At have been carried out at low beam current of alpha particles (50 to $250 \mathrm{nA}$ ), with an integrated beam charge of 100 and $450 \mu \mathrm{C}$, measured with an error smaller than 1-2\% through a Faraday cup connected to a charge integrator. Besides, the reliability of beam charge integrator was checked by thin $\mathrm{Cu}$ monitor foils. In order to produce $\mathrm{NCA}{ }^{211} \mathrm{At} /{ }^{21 \mathrm{~g}} \mathrm{Po}$ for metabolic radiotherapy, a suitable radiochemical separation of At radionuclides from Po by-products and from the Bi target with quality control has been done. The radiochemical separation adopted has been a classical "wet" method based on liquid/liquid extraction.

The $\gamma-$, X-spectra have been measured with coaxial HPGe detectors, the $\alpha$-spectra with Si surface barrier or PIPS detectors, with a resolution of $27 \mathrm{keV}$ (FWHM), and $\beta$-spectra with a conventional liquid scintillation counting LSC and spectrometry system with Horrocks number capability and an higher-resolution liquid scintillation portable spectrometer with $\alpha / \beta$ pulse shape analysis (PSA) discriminator. Significant results from the present measurements and the relevant analysis are shown in the following figures 1-2, concerning gamma-ray and alpha particle spectra referring to the produced radionuclides, including evidence of X-ray emission data for the product ${ }^{211} \mathrm{At} /^{21 \textrm{g}}$ Po.

The radionuclidic purity of the different radiochemistry fractions produced from $\mathrm{Bi}$ target, ${ }^{210} \mathrm{Po}$ impurities and the final solution were also determined accordingly.

In table 1 the experimental thick target yield TTY obtained at LASA Labs is compared with the calculated one (from 28.8 down to $20 \mathrm{MeV}$ ), both from the experimental microscopic data and from model prediction through the EMPIRE II code [15]; the discrepancies appear to be reasonable with regard to overall uncertainties, including the ones concerning the model parameterisation.

Concerning the production route of ${ }^{186 \mathrm{~g}} \mathrm{Re}$ by $(\mathrm{p}, \mathrm{n})$ reaction on ${ }^{186} \mathrm{~W}$, a special effort has been brought up in the

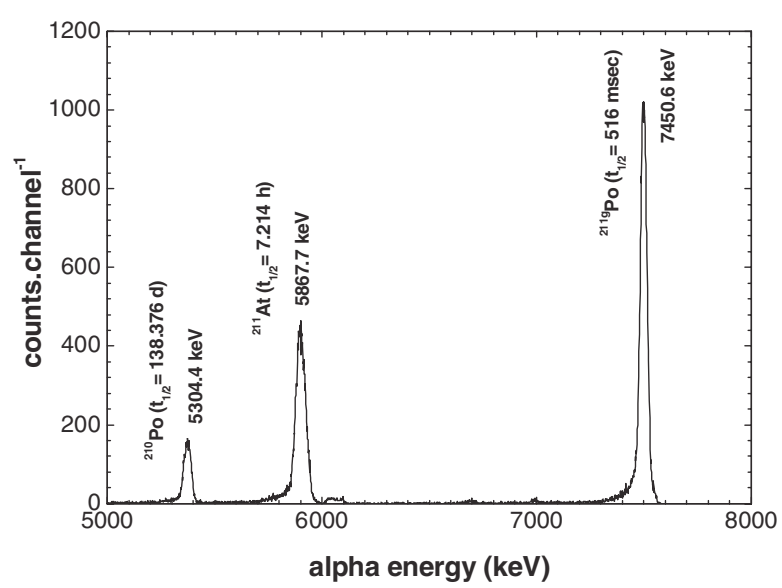

Fig. 2. $\alpha$-spectrum from Bi irradiated with $32.8 \mathrm{MeV} \alpha$-particles. These $\alpha$-spectra are relevant to the radiochemical purity performance of the final products.

Table 1. Experimental TTY $\left(\mathrm{MBq} \cdot \mathrm{C}^{-1}\right)$ measured in this work compared with the calculated ones obtained by integration of existing microscopic measurements fitting and of the model prediction by EMPIRE II code.

\begin{tabular}{|l|l|l|l|l|l|}
\hline $\begin{array}{l}1^{\text {st }} \text { Irrad. } \\
28.8 \mathrm{MeV}\end{array}$ & $\begin{array}{l}\text { experimental } \\
\text { II TTY }\end{array}$ & $\begin{array}{l}\text { by microscopic } \\
\text { measurem. } \\
\text { TTY }\end{array}$ & $\begin{array}{l}\text { discr. } \\
\text { from } \\
\text { exper. } \%\end{array}$ & $\begin{array}{l}\text { EMPIRE } \\
\text { TTY }\end{array}$ & $\begin{array}{l}\text { discr. } \\
\text { from } \\
\text { exper. \% }\end{array}$ \\
\hline${ }^{211} \mathrm{At}$ & $8085 \pm 176$ & 8341 & 3.1 & 9234 & 12.4 \\
\hline
\end{tabular}

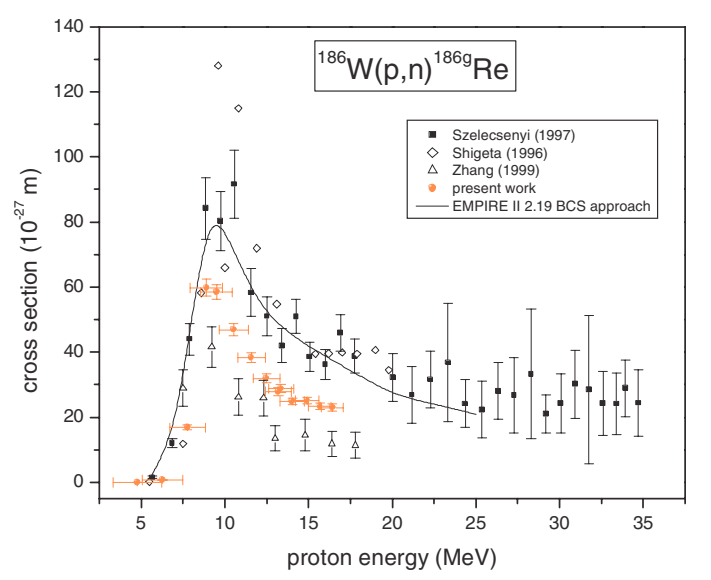

Fig. 3. Experimental cross section data for the ${ }^{186} \mathrm{~W}(\mathrm{p}, \mathrm{n})^{186 \mathrm{~g}} \mathrm{Re}$ reaction and the corresponding theoretical model calculation (full line).

past months at LASA Labs to deduce measured excitation functions starting from irradiation experiments on ${ }^{\text {nat }} \mathrm{W}$ at the above mentioned MC40 cyclotron, with proton beam energy ranging from 7 to $15 \mathrm{MeV}$. In the figure 3 the present results are compared with the previous literature ones and with nuclear model calculated curve obtained through EMPIRE II code [15]. A general comment to figure 3 from our recent results is that our results lie between the previously discrepant experimental data by Szelecsenyi, Shigeta (highest value trend) and Zhang (lowest value trend). The values obtained by the present measurements as from the same figure 3 mostly appear to 


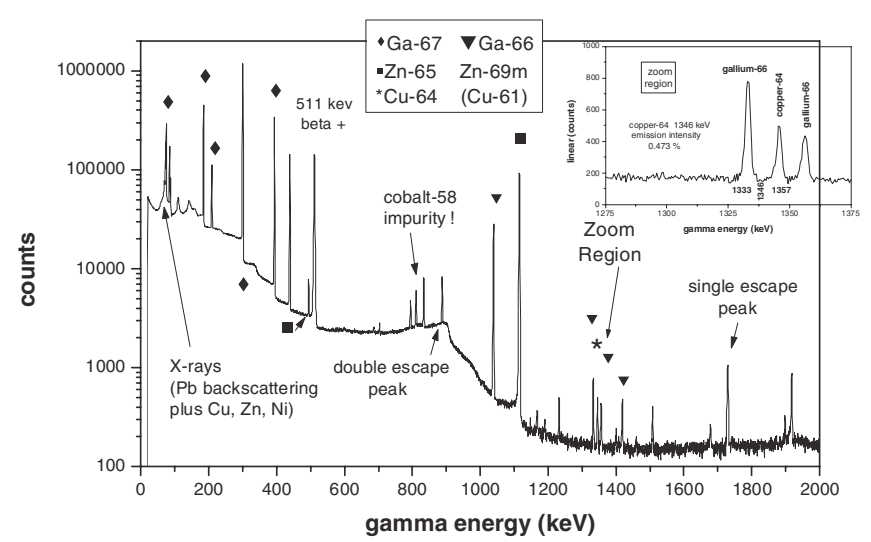

Fig. 4. Significant $\gamma$-line spectroscopic data put in evidence in the energy interval of interest.

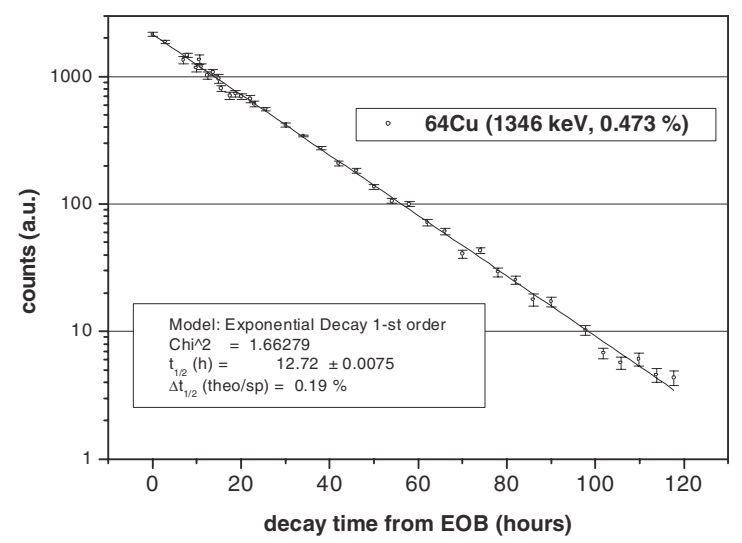

Fig. 5. The decay curve for the $\gamma$ line of reference is given with evidence for the relevant $\chi^{2}$-value.

agree within $3 \sigma$ limits with the other quoted measurements. A discrepancy was noted between the $\gamma$-intensity value adopted by Zhang (9.20\%), our adopted value (8.22\% [14]) and the one adopted by Szelecsenyi ( $8.5 \%)$.

The previous measurements of the excitation function for producing ${ }^{64} \mathrm{Cu}$ have also been revisited, with main concern to gamma spectroscopy and radiation calibration aspects. The results are shown in the figures 4 and 5.

As from the zoom region of the figure 4 the gamma line ( $\alpha=0.473 \%$ [14]) for ${ }^{64} \mathrm{Cu}$, even if very close to the two lines of ${ }^{66} \mathrm{Ga}$, is very well resolved to allow an accurate determination of the ${ }^{64} \mathrm{Cu}$ activity. The reliability of such measurements is confirmed by the $\chi^{2}$-value of the decay curve, obtained from $1346 \mathrm{keV} \gamma$-line as shown in figure 5, then the reliability is reconfirmed of the previously published experimental cross section data [4-6]. The experimental data from the complete experiment are shown in the figure 6 .

\subsection{Nuclear model calculations concerning the relevant excitation functions}

The excitation function calculations have been carried out through the EMPIRE-II code system, by M. Herman [15],

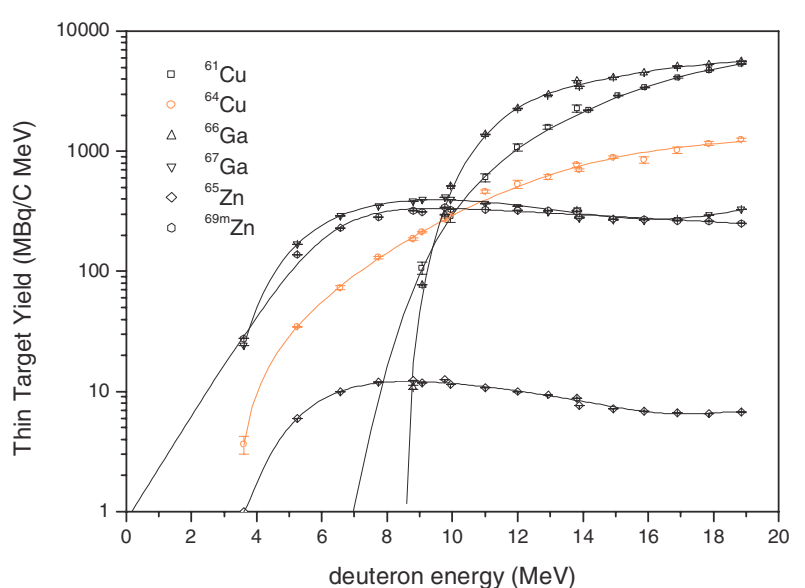

Fig. 6. Experimental thin target yields obtained for the different ${ }^{\text {nat }} \mathrm{Zn}(\mathrm{d}, \mathrm{x})$ nuclear reactions.

accounting for the major reaction mechanisms for the various competing nuclear reaction channels, including the Optical Model (OM) and the full-featured Hauser-Feshbach model. Special care has been devoted to the model parameterisation, especially concerning the nuclear discrete level structure and the level density approach in the continuum, for both target and residual nuclei. When level density parameters could not be determined from the experiments, as for ${ }^{186 g} \mathrm{Re}$, the BCS approach was assumed. Particularly the Monte Carlo Preequilibrium calculations have been successful in approximating the experimental values, in the case of proton induced reaction presently investigated.

Taking advantage from a long-time experience and activities in the field, the excitation functions were calculated for $(\alpha, 2 \mathrm{n})$ and $(\alpha, 3 \mathrm{n})$ reactions on ${ }^{209} \mathrm{Bi}$ target for the production of ${ }^{211}$ At and ${ }^{210} \mathrm{At}$, and for ${ }^{186} \mathrm{~W}(\mathrm{p}, \mathrm{n})$ reaction and ${ }^{103} \mathrm{Rh}(\mathrm{d}, 2 \mathrm{n})$ reaction for producing respectively ${ }^{186 g} \mathrm{Re}$ and ${ }^{103} \mathrm{Pd}$ therapeutic radionuclides and for the ${ }^{232} \mathrm{Th}(\mathrm{p}, 3 \mathrm{n})$ reaction producing ${ }^{230} \mathrm{~Pa} /{ }^{230} \mathrm{U}$ therapeutic alpha emitters.

Particularly, in the last two cases, the research was aimed to investigate the possible production optimal conditions, relevant to future Cyclotron irradiations experiments by proton and deuteron beams.

Moreover, it is noted that a specific suitable target on which proton irradiation can lead to alpha emitters is thorium, especially through the following reaction:

$$
\begin{gathered}
{ }^{232} \mathrm{Th}(\mathrm{p}, 3 \mathrm{n}){ }^{230} \mathrm{~Pa} \stackrel{17.4 \mathrm{~d}, \beta^{-}(8.4 \%)}{\longrightarrow} 230 \mathrm{U} \stackrel{20.8 \mathrm{~d}, \alpha}{\longrightarrow} \\
Q=-13.65 \mathrm{MeV} .
\end{gathered}
$$

Due to the Q-value of this reaction, it is appropriate to utilize the Ispra accelerator, as it can deliver protons up to $38 \mathrm{MeV}$, or dedicated $30 \mathrm{MeV}$ cyclotrons.

Model calculation by EMPIRE II code have been done for other proton induced reactions on ${ }^{232} \mathrm{Th}$ (as possible alternatives for therapeutic radionuclide production, as proposed in [13]) resulting to much lower cross section values, i.e., in the range of few $\mathrm{mb}$. 


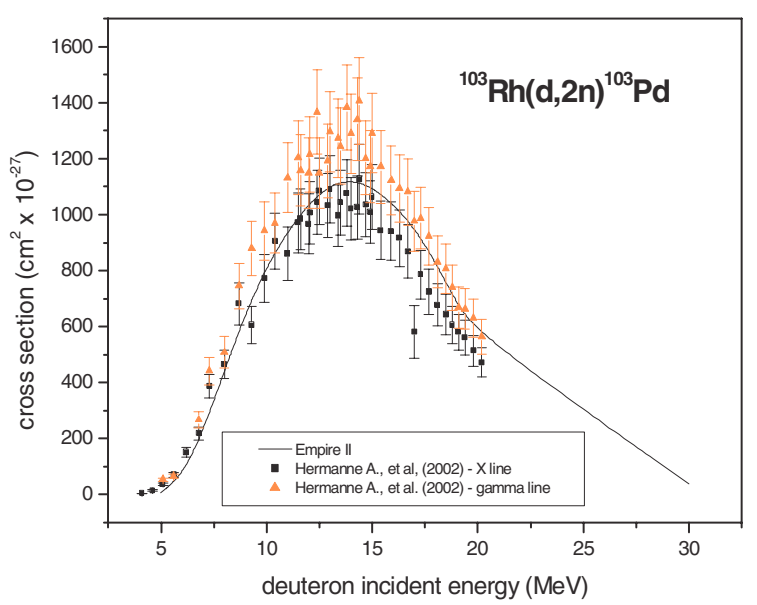

Fig. 7. Experimental cross section data taken from the quoted literature for ${ }^{103} \mathrm{Rh}(\mathrm{d}, 2 \mathrm{n}){ }^{103} \mathrm{Pd}$ reaction and the corresponding theoretical model calculation (full line).

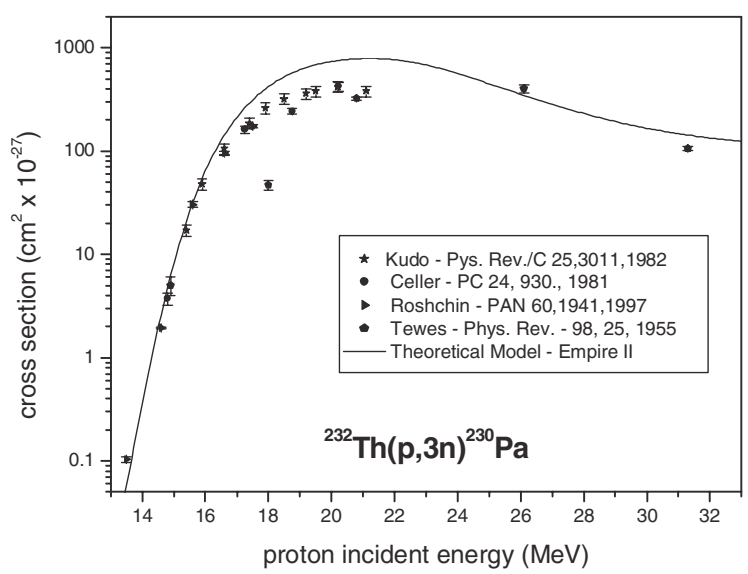

Fig. 8. Experimental data from the literature are compared with the theoretical prediction by EMPIRE II code calculations. Note the significant cross section value of about $300 \mathrm{mb}$ around $20 \mathrm{MeV}$.

A reasonable agreement between the theoretical results and the existing experimental data is shown in the corresponding figures 3,7 and 8 of this paper.

\section{General Remarks}

Our previous results were partially compared with the ones discussed in the context of IAEA CRP on the nuclear data for therapeutic radionuclide production.
Such a comparison gave reason for further investigation as by the present work.

The present research work was funded in the mainframe of the experiment RENIO of the Commission V of INFN and the MIUR, Italy. Thanks are due to JRC-Ispra (EC) Cyclotron Laboratory for irradiating the relevant samples.

\section{References}

1. E. Menapace, C. Birattari, M.L. Bonardi, F. Groppi, Rad. Phys. Chem. 71, 943 (2004).

2. E. Menapace, C. Birattari, M.L. Bonardi, F. Groppi, S. Morzenti, C. Zona, American Institute of Physics 769, 1638 (2005).

3. C. Birattari, M.L. Bonardi, F. Groppi, L. Gini, C. Mainardi, A. Ghioni, G. Ballarini, E. Menapace, K. Abbas, U. Holzwarth, M.F. Stroosnijder, J. Radioan. Nucl. Chem. 257, 229 (2003), Proc. ICI4 Conference (2002).

4. F. Groppi, C. Birattari, M. Bonardi, H.S. Mainardi, E. Menapace, Internatinal Conference on Isotopic and Nuclear Analytical Techniques for Health and Environment, IAEA, Vienna, Austria, IAEA-CN-103/108 (2003).

5. F. Groppi, M.L. Bonardi, E. Menapace, S. Morzenti, C. Zona, L. Canella, Z.B. Alfassi, Nucl. Instrum. Meth. A 562, 1072 (2006).

6. F. Groppi, M. Bonardi, C. Birattari, L. Gini, C. Mainardi, E. Menapace, K. Abbas, U. Holzwarth, R.M.F. Stroosnijder, Nuc. Instrum. Meth. B 213C, 373 (2004).

7. M.L. Bonardi, F. Groppi, H.S.C. Mainardi, V.M. Kokhanyuk, E.V. Lapshina, M.V. Mebel, B.L. Zhuikov, J. Radioanal. Nucl. Chem. 264-1, 101 (2005).

8. A. Alfarano, K. Abbas, U. Holzwarth, M. Bonardi, F. Groppi, Z. Alfassi, E. Menapace, P.N. Gibson, J. Phys.: Conference Series, 41-1, 115 (2006).

9. F. Tarkanyi, S. Takacs, F. Ditroi, A. Hermanne, M. Sonck, Yu. Shubin, Nucl. Instrum. Meth. B 217, 531 (2004).

10. F. Groppi, M.L. Bonardi, C. Birattari, E. Menapace, K. Abbas, U. Holzwarth, A. Alfarano, S. Morzenti, C. Zona, Z.B. Alfassi, Appl. Radiat. Isot. 63, 621 (2005).

11. A. Hermanne, F. Tarkanyi, S. Takàcs, Z. Szucs, Yu.N. Shubin, A.I. Dityuk, Appl. Radiat. Isot. 63, 1 (2005).

12. F. Tarkanyi, S. Takacs, F. Szelecsenyi, F. Ditroi, A. Hermanne, M. Sonck, Nucl. Instrum. Meth. B 252, 160 (2006).

13. Z.B. Alfassi, M.L. Bonardi, F. Groppi, E. Menapace, J. Radioanal. Nucl. Chem. 270-2, 483 (2006).

14. R.B. Firestone, C.M. Baglin, F.S.Y. Chu, Table of Isotopes, 8th edn. (John wiley \& Sons, New York, USA, 1998), Update on CD-ROM, ISBN: 0-471-24699-9.

15. M. Herman, EMPIRE-II Statistical model code for nuclear reaction calculations, version 2.18 and 2.19. 